Research in Astron. Astrophys. 2019 Vol. 19 No. 2, 23(6pp) doi: 10.1088/1674-4527/19/2/23

http://www.raa-journal.org $\quad$ http://www.iop.org/journals/raa

(LTEX: 1902Zarka ms4306.tex; printed on April 3, 2019; 0:34)

$\boldsymbol{R}$ esearch in

Astronomy and

Astrophysics

\title{
Detecting exoplanets with FAST?
}

\author{
Philippe Zarka ${ }^{1}$, Di Li ${ }^{2,3,4}$, Jean-Mathias Grießmeier ${ }^{5}$, Laurent Lamy ${ }^{1}$, Julien N. Girard ${ }^{6}$, \\ Sébastien L. G. Hess ${ }^{7}$, T. Joseph W. Lazio ${ }^{8}$ and Gregg Hallinan ${ }^{9}$ \\ 1 LESIA, CNRS - Observatoire de Paris, PSL 92190, Meudon, France; philippe.zarka@obspm.fr \\ 2 National Astronomical Observatories, Chinese Academy of Science, Beijing 100101, China; \\ dili@nao.cas.cn \\ 3 CAS Key Laboratory of FAST, National Astronomical Observatories, Chinese Academy of \\ Sciences, Beijing 100101, China \\ 4 School of Astronomy and Space Science, University of Chinese Academy of Sciences, Beijing \\ 101408, China \\ 5 LPC2E, CNRS - Université d'Orléans, 45071, Orléans, France \\ 6 Université Paris VII, AIM/Irfu/SAp/CEA-Saclay, Orme des Merisiers, 91190, Gif-Sur-Yvette, \\ France \\ 7 ONERA - DESP, 31000, Toulouse, France \\ 8 JPL - California Institute of Technology, Pasadena, CA 91109, USA \\ 9 California Institute of Technology, Pasadena, CA 91125, USA
}

Received 2018 February 6; accepted 2018 June 5

\begin{abstract}
We briefly review the various proposed scenarios that may lead to nonthermal radio emissions from exoplanetary systems (planetary magnetospheres, magnetosphereionosphere and magnetosphere-satellite coupling, and star-planet interactions), and the physical information that can be drawn from their detection. The latter scenario is especially favorable to the production of radio emission above $70 \mathrm{MHz}$. We summarize the results of past and recent radio searches, and then discuss FAST characteristics and observation strategy, including synergies. We emphasize the importance of polarization measurements and a high duty-cycle for the very weak targets that radio-exoplanets prove to be.
\end{abstract}

Key words: plasmas — radiation mechanisms: non-thermal — methods: observational - telescopes (radio) — planets and satellites: magnetic fields — radio continuum: planetary systems

\section{INTRODUCTION}

Planets are the most favorable cradle of life. As of today, nearly 4000 exoplanets are known (exoplanet.eu - mainly by radial velocity or transit measurements, from which masses, orbital parameters, sizes, densities and atmospheric compositions can be inferred). In our solar system, magnetized planets are strong radio sources (Jupiter is as bright as the Sun at decameter wavelengths). Radio detection of exoplanets aims at the physical characterization of exoplanets and comparative studies with solar system planets. 


\section{EXPERIENCE FROM SOLAR SYSTEM PLANETS AND THEORY}

There are six magnetized planets in the solar system with a planetary-scale magnetic field: Mercury, Earth, Jupiter, Saturn, Uranus and Neptune. In their magnetospheres, various processes accelerate electrons to $\mathrm{keV}-\mathrm{MeV}$ energies, leading to high-latitude (auroral) radio emissions (Zarka 1998), whose spectra are displayed in Figure 11 The corresponding radio sources have been studied remotely and in situ (e.g. Huff et al. 1988; Treumann \& Pottelette 2002; Lamy et al. 2010). The emissions were found to be coherent cyclotron (maser) radiation from $\mathrm{keV}$ electrons. The emission frequency depends on the local cyclotron frequency, which is proportional to the magnetic field amplitude, and is thus generally below a few 10 s of MHz, reaching $40 \mathrm{MHz}$ for Jupiter. Emissions are very intense (brightness temperature up to $10^{15-20} \mathrm{~K}$ ), sporadic (bursts lasting from msec to hours), anisotropic (beamed at large angles from the magnetic field) and circularly polarized (right-handed in northern magnetic hemispheres and lefthanded in southern ones) (Wu 1985; Zarka 1998; Treumann 2006; Hess et al. 2008). Weaker incoherent synchrotron emission is generated by MeV electrons in Jupiter's radiation belts (de Pater 1990), which was recently imaged with LOFAR (Girard et al. 2016).

The energy drivers for electron acceleration up to $\mathrm{keV}$ energies include (Zarka et al. 2001; Zarka 2007, 2017; Nichols 2011):

- Stellar wind-magnetosphere interaction (super-Alfvénic, via compressions and reconnections),

- Magnetosphere-ionosphere coupling,

- Magnetosphere-satellite coupling (sub-Alfvénic, via reconnection or unipolar inductor interaction),

- Star-planet interaction (SPI - sub-Alfvénic, via reconnection or unipolar inductor interaction).

\section{PREDICTIONS FOR EXOPLANETS}

Jupiter's decametric emission is detectable at no more than $0.2 \mathrm{pc}$ over the Galactic background. Thus, the search for exoplanetary radio emissions must had relied on scaling laws and extrapolations, as well as more direct theoretical predictions.

Study of solar wind-magnetosphere interactions led Zarka et al. (2001) and Zarka (2007, 2010) to propose that a planet's low-frequency radio output is proportional to kinetic (including CME - coronal mass ejection) and magnetic (Poynting flux) power inputs on the obstacle's cross-section. Extrapolation to hot Jupiters led to prediction of radio fluxes up to $10^{3-5}$ times that of Jupiter (Fig. 2). The IoJupiter electrodynamic interaction transposed to plasma SPI led the same authors to predict radio outputs proportional to the Poynting flux input, up to $10^{6}$ times Jupiter's (Fig. 22).

Nichols (2011, 2012), based on the physics of magnetosphere-ionosphere interaction, predicted a large low-frequency radio output, up to $10^{4}$ times Jupiter's, for fast rotating planets orbiting stars with a bright ultraviolet (UV)-X-ray luminosity. Willes \& Wu (2004, 2005) extended the theoretical frame of the Io-Jupiter unipolar interaction to terrestrial planets around white dwarfs, predicting a large radio output at frequencies $\gg 1 \mathrm{GHz}$.

In all cases, detection is the difficult step. Even if star-planet systems will be unresolved in radio, subsequent discrimination between stellar and planetary emission will rely (easily) on the polarizations (circular for planets) and periodicities (rotation, orbital) of the detected radio emission.

\section{MOTIVATIONS FOR STUDYING RADIO EMISSIONS FROM EXOPLANETS}

Magnetospheric radio emissions provide unique information on planetary magnetic field amplitude and tilt (Jupiter's magnetic field was first measured that way (Burke \& Franklin 1955)), and thus on the planetary dynamo, giving constraints on the planetary interior structure. It is also a signature of planetary rotation, due to the emission anisotropy (the rotation periods of Jupiter (Higgins et al. 1997), Saturn 


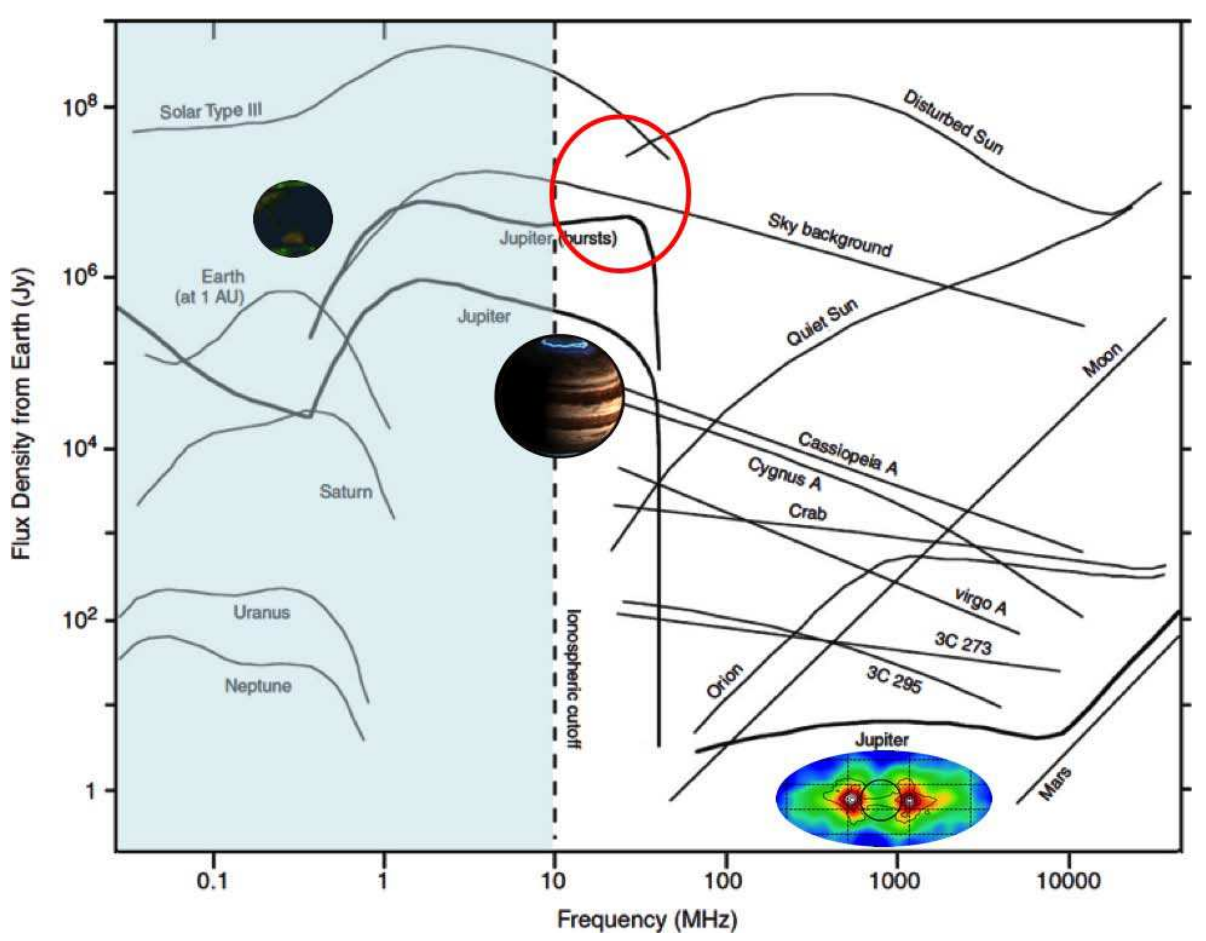

Fig. 1: Spectra of astronomical radio sources detected in the Earth's vicinity. Auroral planetary spectra lie below the Earth's ionospheric cut-off, except Jupiter's decametric-to-decimetric emissions. Normalized to the same observer distance of 1 AU, Jupiter's spectrum must be upscaled by $\times 20$, Saturn's by $\times 100$, Uranus' by $\times 400$ and Neptune's by $\times 900$, so that all are grouped within $2-3$ orders of magnitude.

(Desch \& Kaiser 1981), Uranus and Neptune were measured via their radio emission), that will permit directly testing exoplanetary spin-orbit locking. It may reveal the presence of satellites (e.g. Bigg 1964) interacting with the planetary magnetic field. Hess \& Zarka (2011) showed that radio emissions also allow researchers to probe planetary orbit inclination, and SPI and magnetospheric dynamics at large.

A magnetosphere also shields a planet's atmosphere and surface against cosmic rays, stellar wind and CME bombardment, preventing $\mathrm{O}_{3}$ destruction and atmospheric erosion or escape. It is thus a favorable condition for life (Grießmeier et al. 2004; Lammer et al. 2006). Finally, radio emission detection may evolve as an independent discovery tool for planets around active, magnetic or variable stars.

\section{PAST AND ONGOING OBSERVATIONS AND RESULTS}

Targeted searches have been guided by theory and scaling laws applied to the exoplanet census (with e.g. $\tau$ Boo, $v$ And or $55 \mathrm{Cnc}$ as good candidates (Lazio et al. 2004; Grießmeier et al. 2007), strongly magnetized stars (such as HD 189733 (Donati et al. 2006)), planets with very elliptical orbits and closein periastrons (such as HD 80606 (Lazio \& Farrell 2007; Lazio et al. 2010)) and systems with possible optical SPI signatures (such as HD 179949 (Shkolnik et al. 2008) and references therein). Observations were conducted with the VLA at $\geq 74 \mathrm{MHz}$ (Farrell et al. 2003, 2004; Lazio \& Farrell 2007), UTR2/Kharkov in the range 10-32 MHz (Ryabov et al. 2004) and the GMRT at $\geq 150 \mathrm{MHz}$ (Hallinan et al. 2013; Lecavelier des Etangs et al. 2013). No confirmed detection has been reached. A hint of a radio occultation of Hat-P-11 b by its star was found by GMRT, but in only one of two observations with similar geometry, thus it remains to be confirmed (Lecavelier des Etangs et al. 2013). Ongoing targeted 


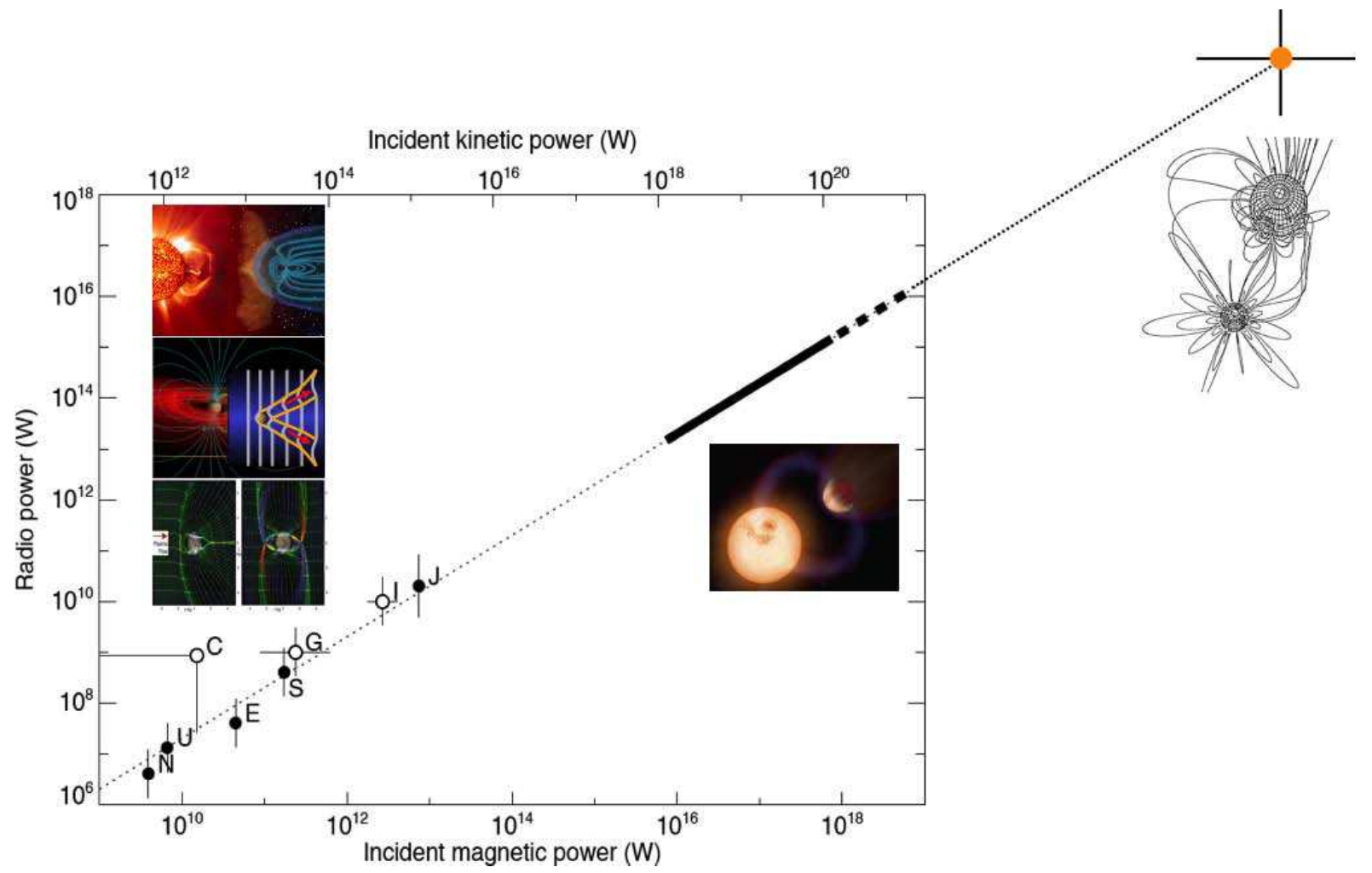

Fig. 2: Scaling law relating magnetospheric (Earth, Jupiter, Saturn, Uranus and Neptune) and satelliteinduced (Io, Ganymede and Callisto) radio power to incident Poynting flux of the plasma flow on the obstacle. The dashed line has a slope of 1 , emphasizing the proportionality between incident power and outgoing radio power with a coefficient of $2 \times 10^{3}$. The thick bar extrapolates the magnetospheric interaction (solid) and satellite-planet electrodynamic interactions (dashed) to hot Jupiters. The orange dot indicates the case of the RS CVn magnetic binary V711 $\tau$ discussed in Zarka (2010); Zarka et al. (2015a). The insets illustrate the types of interaction.

observations include campaigns at UTR-2 (10-32 MHz, $\geq 100 \mathrm{~h})$, programs with LOFAR in cycles 0 to 8 (20-80 MHz, for a total of $\sim 170 \mathrm{~h}$ ), and a program with the Long Wavelength Array (LWA) that plans to observe for $\sim 5000 \mathrm{~h}$.

In parallel, correlations of the exoplanet catalog at exoplanet.eu with low-frequency surveys open new perspectives. Four candidates were found in the GMRT TGSS $150 \mathrm{MHz}$ survey, at the 10-100 mJy level, out of 175 exoplanetary systems in the surveyed field (Sirothia et al. 2014), but these cases remain unconfirmed. Analysis of LOFAR surveys is ongoing, with Multi-Frequency Snapshot Sky Survey in the $160 \mathrm{MHz}$ band, at $\sim 5 \mathrm{mJy}$ sensitivity, and later in the $30-75 \mathrm{MHz}$ band at $\sim 15 \mathrm{mJy}$ sensitivity from the LOFAR deep surveys program (Shimwell et al. 2017; Loh et al. in preparation). Permanent all-sky observations at $\sim 1 \mathrm{~s}$ resolution have started at the Owens Valley Long Wavelength Array (OLWA).

As a preliminary conclusion, radio emissions much stronger than Jupiter's at frequencies $\geq 150 \mathrm{MHz}$ appear to be rare, which could be due to a too low planetary magnetic field, emission beaming out of the observer's line of sight at the time of the observations or too weak flux density. 


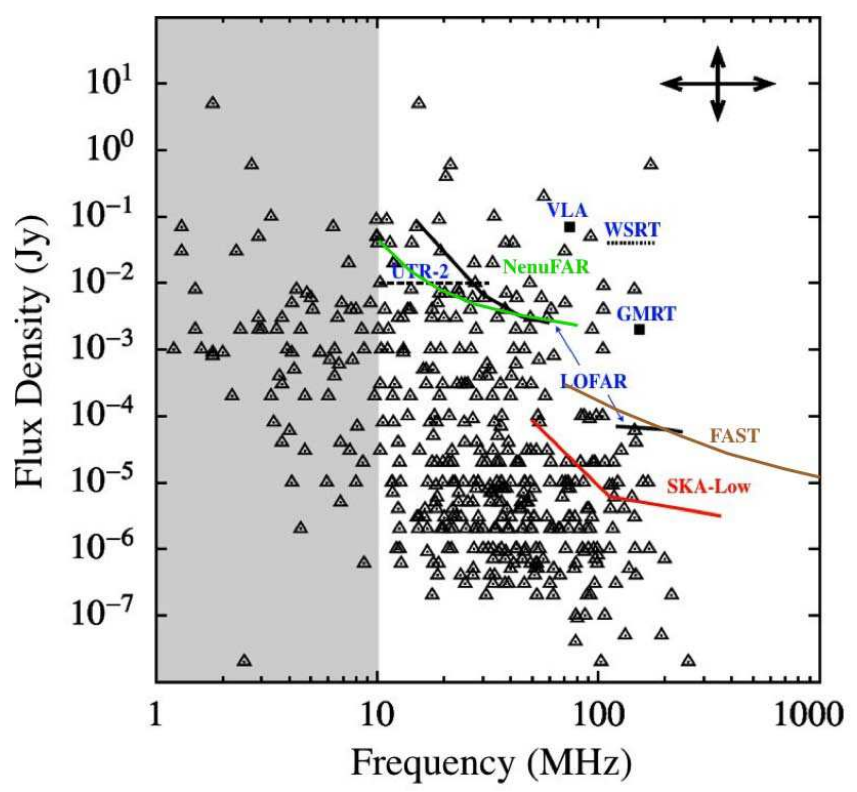

Fig. 3: Predicted maximum emission frequency and expected radio flux for known exoplanets (in 2011, indicated by triangle symbols) for a rotation-independent planetary magnetic field and the scaling law displayed in Figure 2 The approximate sensitivities of several instruments are shown (for $1 \mathrm{~h}$ of integration time and a bandwidth of $4 \mathrm{MHz}$ ). Frequencies below $10 \mathrm{MHz}$ are not observable from the ground (ionospheric cut-off); adapted from (Grießmeier et al. 2011). More recent observations have populated this diagram, but only $\sim$ doubling the number of FAST candidates.

Table 1: FAST Characteristics at Frequencies below L-band

\begin{tabular}{lcccccc}
\hline $\begin{array}{l}\text { Frequency } \\
(\mathrm{MHz})\end{array}$ & $\begin{array}{c}T_{\text {sky }} \\
(\mathrm{K})\end{array}$ & $\begin{array}{c}T_{\text {sys }} \\
(\mathrm{K})\end{array}$ & $\begin{array}{c}\text { SEFD } \\
(\mathrm{Jy})\end{array}$ & $\begin{array}{c}\text { Sensitivity }[4 \mathrm{MHz} \times 1 \mathrm{~h}, \text { polarized] } \\
(\mathrm{mJy})\end{array}$ & $\begin{array}{c}\text { Confusion }{ }^{a}[\text { unpolarized] } \\
(\mathrm{mJy})\end{array}$ & $\begin{array}{c}\text { Angular resolution } \\
(\operatorname{arcmin})\end{array}$ \\
\hline 70 & 2500 & 100 & 110 & 0.5 & 3100 & 50 \\
140 & 420 & 80 & 22 & 0.1 & 480 & 25 \\
280 & 72 & 40 & 5 & 0.020 & 73 & 13 \\
560 & 13 & 10 & 1 & 0.004 & 11 & 7 \\
1150 & 5 & 10 & 0.7 & 0.003 & 1.6 & 3 \\
\hline
\end{tabular}

Notes: ${ }^{a}$ Theoretical estimate from Condon (2005).

\section{SCIENCE OUTCOME ENABLED BY FAST}

Following the previous conclusion, it is necessary to explore a large sample of targets with the highest possible continuum sensitivity, preferably at low frequencies, with circular polarization or full Stokes observations, down to the thermal noise level. Multi-epoch observations with integration times of a few hours at each epoch are required for reaching a good sensitivity and addressing time variations of intrinsically (or beaming-induced) sporadic emissions.

The FAST radio telescope (Nan et al.2011) can observe down to $70 \mathrm{MHz}$, within a broad frequency range, with full polarization measurements. Table 1 summarizes its relevant characteristics for SPI studies. Comparing the sensitivities with the typical flux densities of Jupiter's bursts at $30-40 \mathrm{MHz}$, about $40 \mu \mathrm{Jy}$ at a $10 \mathrm{pc}$ range, it appears that FAST has the sensitivity to detect moderate intensity exoplanetary emissions, provided that the frequency of emission exceeds $70 \mathrm{MHz}$. A frequency $>70 \mathrm{MHz}$ corre- 
sponds to cyclotron emission from a source with a magnetic field amplitude $>25 \mathrm{G}$. Figure 3 shows the FAST sensitivity (as well as that of other low-frequency instruments) compared to the predicted maximum emission frequency and expected radio flux for known exoplanets in 2011, extrapolated following Grießmeier et al. (2007). Only a few candidate exoplanets have a predicted magnetic field reaching or exceeding 25 G. FAST will thus likely be best adapted to search for SPI emissions (exoplanet-induced as proposed by Zarka (2007, 2017), including for terrestrial planets around white dwarfs as proposed by Willes \& Wu (2004, 2005)), down to moderate intensities, whereas magnetospheric exoplanetary emissions will be rather the target of lower frequency radio arrays such as LOFAR (van Haarlem et al. 2013) and NenuFAR (Zarka et al. 2012, 2015b).

Due to the relatively low angular resolution of FAST at low frequencies, exoplanet detection via direct imaging will be severely limited by confusion, i.e. spatial noise across the sky background. The possible ways to lower this limit are (i) observing in circular polarization - for which confusion noise is expected to be much smaller than for unpolarized emission - or full Stokes, and (ii) looking for time variable emission in well-defined fields. The latter method is favored by FAST's constant beam size in all pointing directions (Nan et al. 2011) and a frequency range well above the ionospheric cut-off $(\sim 10 \mathrm{MHz})$.

A key element of the success of FAST in exoplanet radio search and study will be the possibility to conduct a large number of multi-epoch targeted observations as well as extensive polarized surveys (e.g. of all observable stellar systems up to a distance of $10 \mathrm{pc}: \sim 200$ known stars and $\sim 35$ currently known exoplanets). One drawback is the availability of a single beam at low frequencies, which will require a strong programmatic decision in favor of exoplanet studies in order to perform a significant program (such as the LWA - HJUDE program). Follow-up observations of targets identified by other instruments (LOFAR, GMRT) also offer good prospects (FAST covers a large declination range in common with these two facilities).

\section{SYNERGIES}

Advantage will be taken of synergies between radio observations of exoplanets and of stellar solar-like bursts. Stellar flares could be planet induced or intrinsic: GHz periodic bright pulses with periods $\sim 2 \mathrm{~h}$ and $100 \%$ circularly polarized have been found by Hallinan et al. (2007, 2008, 2015) and attributed to cyclotron masers in a brown dwarf with magnetic field $\sim 2 \mathrm{kG}$. Further, there is a possibility to explore targets ranging from brown dwarfs to exoplanets (faster rotation, cooler and more neutral atmosphere, with larger-scale stable magnetic field topologies and weaker field amplitude). Tracing magnetic fields and radio flux densities from brown dwarfs to planets will introduce unique constraints for dynamo theories and radio emission scaling laws. Lower mass planets seem to be more frequent around $\mathrm{M}$ dwarfs, with close-in planets lying in the habitable zone (Dressing \& Charbonneau 2013). Commensal SETI searches would be possible with adequate instrumentation (very high spectral resolution of waveform measurements).

Synergies will also exist with observations at other wavelengths:

- with radio observations at frequencies below $70 \mathrm{MHz}$ (LOFAR, NenuFAR, UTR-2),

- with Zeeman Doppler Imaging (CFHT/Espadons, TBL/Narval, CFHT/Spirou) permitting stellar magnetic field measurements and planet searches around M dwarfs (Fares et al. 2010),

- with UV-X-ray observations (HST, JWST, XMM-Newton, Chandra, Athena) of stellar flares and exoplanet atmospheres,

- with PLATO and TESS revealing more nearby exoplanets, and ESO-VLT/NGTS, ESPRESSO or Gaia surveys that will provide tens of exoplanets per field-of-view or a few degrees. 


\section{CONCLUSIONS}

Study of radio emissions from exoplanets and the star-planet connection is a broad new field to explore. Its theoretical frame is ready. There are currently optimistic prospects with LOFAR, and its giant compact extension NenuFAR. The advent of SKA will enable a sensitivity improvement of $\sim 30 \times$ compared to LOFAR, down to the $10 \mu \mathrm{Jy}$ level, at frequencies $\geq 50 \mathrm{MHz}$. This makes it highly likely that SKA-Low will detect exoplanetary radio signals (Zarka et al. 2015a). But this will happen in 2020+, and FAST, being operational now, benefits from a favorable window for exoplanet and SPI radio search. It will also cover a declination range complementary to that of LOFAR and NenuFAR (mostly the northern hemisphere), and SKA (mostly the southern hemisphere) and be compatible with that of the GMRT.

Acknowledgements This work is supported by the National Key R\&D Program No. 2017YFA0402600, the CAS International Partnership Program No. 14A11KYSB20160008, and the NSFC grant No. 11725313. Part of this research (TJWL) was carried out at JPL/Caltech under a contract with NASA.

\section{References}

Bigg, E. K. 1964, Nature, 203, 1008

Burke, B. F., \& Franklin, K. L. 1955, J. Geophys. Res., 60, 213

Condon, J. J. 2005, in Astronomical Society of the Pacific Conference Series, 345, From Clark Lake to the Long

Wavelength Array: Bill Erickson's Radio Science, eds. N. Kassim, M. Perez, W. Junor, \& P. Henning, 237

de Pater, I. 1990, ARA\&A, 28, 347

Desch, M. D., \& Kaiser, M. L. 1981, Geophys. Res. Lett., 8, 253

Donati, J.-F., Howarth, I. D., Jardine, M. M., et al. 2006, MNRAS, 370, 629

Dressing, C. D., \& Charbonneau, D. 2013, ApJ, 767, 95

Fares, R., Donati, J.-F., Moutou, C., et al. 2010, MNRAS, 406, 409

Farrell, W. M., Desch, M. D., Lazio, T. J., Bastian, T., \& Zarka, P. 2003, in Astronomical Society of the Pacific

Conference Series, 294, Scientific Frontiers in Research on Extrasolar Planets, eds. D. Deming, \& S. Seager, 151

Farrell, W. M., Lazio, T. J. W., Desch, M. D., Bastian, T. S., \& Zarka, P. 2004, in IAU Symposium, 213,

Bioastronomy 2002: Life Among the Stars, eds. R. Norris \& F. Stootman, 73

Girard, J. N., Zarka, P., Tasse, C., et al. 2016, A\&A, 587, A3

Grießmeier, J.-M., Stadelmann, A., Penz, T., et al. 2004, A\&A, 425, 753

Grießmeier, J.-M., Zarka, P., \& Spreeuw, H. 2007, A\&A, 475, 359

Grießmeier, J.-M., Zarka, P., \& Girard, J. N. 2011, Radio Science, 46, RS0F09

Hallinan, G., Bourke, S., Lane, C., et al. 2007, ApJ, 663, L25

Hallinan, G., Antonova, A., Doyle, J. G., et al. 2008, ApJ, 684, 644

Hallinan, G., Sirothia, S. K., Antonova, A., et al. 2013, ApJ, 762, 34

Hallinan, G., Littlefair, S. P., Cotter, G., et al. 2015, Nature, 523, 568

Hess, S., Mottez, F., Zarka, P., \& Chust, T. 2008, Journal of Geophysical Research (Space Physics), 113, A03209

Hess, S. L. G., \& Zarka, P. 2011, A\&A, 531, A29

Higgins, C. A., Carr, T. D., Reyes, F., et al. 1997, J. Geophys. Res., 102, 22033

Huff, R. L., Calvert, W., Craven, J. D., et al. 1988, J. Geophys. Res., 93, 11445

Lammer, H., Khodachenko, M. L., Lichtenegger, H. I. M., et al. 2006, in European Planetary Science Congress 2006, 388

Lamy, L., Schippers, P., Zarka, P., et al. 2010, Geophys. Res. Lett., 37, L12104

Lazio, W., T. J., Farrell, W. M., Dietrick, J., et al. 2004, ApJ, 612, 511

Lazio, T. J. W., \& Farrell, W. M. 2007, ApJ, 668, 1182

Lazio, T. J. W., Shankland, P. D., Farrell, W. M., \& Blank, D. L. 2010, AJ, 140, 1929

Lecavelier des Etangs, A., Sirothia, S. K., Gopal-Krishna, \& Zarka, P. 2013, A\&A, 552, A65 
Nan, R., Li, D., Jin, C., et al. 2011, International Journal of Modern Physics D, 20, 989

Nichols, J. D. 2011, MNRAS, 414, 2125

Nichols, J. D. 2012, MNRAS, 427, L75

Ryabov, V. B., Zarka, P., \& Ryabov, B. P. 2004, Planet. Space Sci., 52, 1479

Shimwell, T. W., Röttgering, H. J. A., Best, P. N., et al. 2017, A\&A, 598, A104

Shkolnik, E., Bohlender, D. A., Walker, G. A. H., \& Collier Cameron, A. 2008, ApJ, 676, 628

Sirothia, S. K., Lecavelier des Etangs, A., Gopal-Krishna, Kantharia, N. G., \& Ishwar-Chandra, C. H. 2014, A\&A, 562, A 108

Treumann, R. A. 2006, A\&A Rev., 13, 229

Treumann, R. A., \& Pottelette, R. 2002, Advances in Space Research, 30, 1623

van Haarlem, M. P., Wise, M. W., Gunst, A. W., et al. 2013, A\&A, 556, A2

Willes, A. J., \& Wu, K. 2004, MNRAS, 348, 285

Willes, A. J., \& Wu, K. 2005, A\&A, 432, 1091

Wu, C. S. 1985, Space Sci. Rev., 41, 215

Zarka, P. 1998, J. Geophys. Res., 103, 20159

Zarka, P. 2007, Planet. Space Sci., 55, 598

Zarka, P., Treumann, R. A., Ryabov, B. P., \& Ryabov, V. B. 2001, Ap\&SS, 277, 293

Zarka, P. 2010, in Astronomical Society of the Pacific Conference Series, 430, Pathways Towards Habitable Planets, eds. V. Coudé du Foresto, D. M. Gelino, \& I. Ribas, 175

Zarka, P., Girard, J. N., Tagger, M., \& Denis, L. 2012, in SF2A-2012: Proceedings of the Annual Meeting of the French Society of Astronomy and Astrophysics, eds. S. Boissier, P. de Laverny, N. Nardetto, et al., 687

Zarka, P., Lazio, J., \& Hallinan, G. 2015, Advancing Astrophysics with the Square Kilometre Array (AASKA14), 120

Zarka, P., Tagger, M., Denis, L., et al. 2015b, in International Conference on Antenna Theory and Techniques (ICATT), Kharkiv, Ukraine, 13

Zarka, P. 2017, Star-Planet Interactions in the Radio Domain: Prospect for Their Detection, Handbook of Exoplanets (Springer Living Reference Work), 22 